\title{
Miranda
}

Revue pluridisciplinaire du monde anglophone /

Multidisciplinary peer-reviewed journal on the English-

speaking world

$12 \mid 2016$

Mapping gender. Old images ; new figures

\section{Claire Parfait et Marie-Jeanne Rossignol (dir.), « Ecrire l'esclavage », Revue du Philanthrope 5 (2014)}

\section{Bertrand Van Ruymbeke}

\section{OpenEdition}

\section{Journals}

\section{Electronic version}

URL: http://journals.openedition.org/miranda/7881

DOI: 10.4000/miranda.7881

ISSN: 2108-6559

Publisher

Université Toulouse - Jean Jaurès

\section{Electronic reference}

Bertrand Van Ruymbeke, "Claire Parfait et Marie-Jeanne Rossignol (dir.), « Ecrire l'esclavage », Revue du Philanthrope 5 (2014)", Miranda [Online], 12 | 2016, Online since 26 February 2016, connection on 16 February 2021. URL: http://journals.openedition.org/miranda/7881 ; DOI: https://doi.org/10.4000/ miranda.7881

This text was automatically generated on 16 February 2021

\section{(c) $(1)$}

Miranda is licensed under a Creative Commons Attribution-NonCommercial-NoDerivatives 4.0 International License. 


\title{
Claire Parfait et Marie-Jeanne Rossignol (dir.), « Ecrire l'esclavage ", Revue du Philanthrope 5 (2014)
}

\author{
Bertrand Van Ruymbeke
}

\section{REFERENCES}

Claire Parfait et Marie-Jeanne Rossignol (dir.), « Ecrire l'esclavage », Revue du Philanthrope 5 (2014), 200 p, ISSN 2272-8147

1 La Revue du Philanthrope, Histoire et Mémoires de la traite négrière et de leurs abolitions en Normandie, nous offre un numéro des plus intéressants et des plus agréables à lire intitulé «Ecrire sur l'esclavage» et coordonné par Claire Parfait et Marie-Jeanne Rossignol. Ces articles sont issus des communications présentées à un colloque du même nom qui s'est tenu au Havre en novembre 2013, dans le cadre du programme de Sorbonne-Paris-Cité, «Ecrire l'histoire depuis les marges: le cas des AfricainsAméricains ». Soulignons l'efficacité d'une publication en une année à peine. Ce numéro est un mélange, heureux et équilibré, entre à la fois des réflexions sur le récit d'esclave, en anglais slave narrative, comme genre et comme source pour l'histoire des Amériques et de l'espace atlantique et des études de cas sur des itinéraires d'esclaves ou des ouvrages, en Amérique du Nord (Etats-Unis et Canada), aux Antilles anglophones (Jamaïque) et hispanophones (Cuba) et au Brésil, ainsi qu'entre des contributions de jeunes chercheurs et de professeurs confirmés. Ajoutons la présence opportune de belles illustrations (photographies, portraits, couvertures d'ouvrage). Enfin, ce numéro contient aussi une recension de la traduction française de l'ouvrage-clé de l'historien américain Marcus Rediker, A bord du négrier. Une histoire atlantique de la traite, publié au Seuil en 2013 (titre original : The Slave Ship. A Human History, 2008). 
2 Voici un numéro de belle facture, à la fois très utile pour l'étudiant et le chercheur. Claire Parfait offre en ouverture une utile mise en perspective qui montre combien histoire et historiographie s'entremêlent dans la production, l'utilisation et l'étude des récits d'esclave étatsuniens des années 1830 à tout récemment. Toujours aux ÉtatsUnis, Michaël Roy étudie les récits d'esclave avant la guerre de Sécession publiés pour des raisons d'abord militantes, pour servir la cause abolitionniste du moment, puis littéraires et enfin commerciales. Lucia Bergamasco étudie le concept de l'honneur, une valeur si centrale du Sud d'avant la guerre de Sécession comme l'ont montré les travaux de l'historien américain Bertram Wyatt-Brown, dans les récits d'esclave, une valeur que partagent les auteurs de ces récits avec les planteurs mais dans sa version nordiste plus éthique que chevaleresque. Comme l'écrit Lucia Bergamasco, «l'honneur des esclaves » se présente comme " un contre-récit de l'honneur sudiste » (50). MarieJeanne Rossignol opportunément rappelle en prenant l'histoire et le récit de Sojourner Truth, une esclave de l'arrière-pays néerlandophone de l'État de New York, que l'esclavage au XVIII et lors du premier XIX ${ }^{\mathrm{e}}$ siècle ne fut hélas pas l'apanage du Sud. Notons à ce propos le titre d'une sous-partie «Cruautés de l'esclavage nordiste » (60). Au-delà du personnage lui-même, cet essai porte sur la transition difficile, trop souvent prise pour acquise, vers une liberté toute relative et certainement pas une citoyenneté pleine et entière suite à l'application des lois sur l'émancipation graduelle des esclaves de 1799 et de 1817. Un essai que complète le suivant, par Claudine Raynaud, plus axé sur le texte de Truth lui-même. Ada Savin nous présente le couple William et Ellen Craft, auteurs d'un récit de fuite qui ont réussi à se faire une place sur la scène du mouvement abolitionniste britannique dans les années 1850 avant de s'installer en Géorgie en 1868. La contribution de Françoise Palleau-Papin présente une étude originale des métamorphoses contemporaines et littéraires des récits d'esclaves. L'essai de Jean-Pierre Le Glaunec apporte une contribution éclairante sur l'absence de récits d'esclaves aux Antilles anglophones et comment (et à partir de quelles sources disponibles) combler cette absence, notamment en s'appuyant sur les très nombreuses annonces publiées dans la presse par les maîtres pour tenter de récupérer des esclaves fugitifs. Trois contributions nous emmènent au Canada, à Cuba et au Brésil pour une étude de textes et d'auteurs moins connus, du moins de ceux qui travaillent sur les États-Unis. Enfin, deux notes portent sur le récit de Solomon Northup, Twelve Years a Slave (1853), sorti de l'oubli en 2013 par le film de Steve McQueen, et sur l'ouvrage Marie ou l'Esclavage aux États-Unis (1835) écrit par le compagnon de voyage de Tocqueville, Gustave de Beaumont, et qui décrit, contrairement à ce que son titre suggère, l'Amérique jacksonienne et non l'esclavage à proprement parler. Ces études de récits parfaitement contextualisées à la fois dans l'espace et le temps et ces vignettes biographiques fascinantes à découvrir ou redécouvrir témoignent opportunément de la richesse et de la vitalité de la recherche française et francophone dans le domaine de l'esclavage aux Amériques, notamment aux États-Unis. 


\section{INDEX}

Mots-clés: esclavage, traite négrière, récits d'esclaves, États-Unis, Sud, Antilles anglophones, Jamaïque, Cuba, Canada, Brésil, guerre de Sécession, abolitionnisme, slave narratives, Reconstruction, New York, manumission, lois d'émancipation, Grand Réveil, Atlanta, Boston, Londres, La Nouvelle-Orléans, marronnage, Toronto, Bénin

Keywords: slavery, slave trade, slave narratives, abolitionism, United States, US South, Civil War, Reconstruction, British Caribbean, Jamaica, Cuba, Canada, Brazil, manumission, emancipation laws, fugitive slaves, New York, Great Awakening, Atlanta, Boston, London, New Orleans, Toronto, Benin

\section{AUTHORS}

\section{BERTRAND VAN RUYMBEKE}

Professeur des Universités

Université de Paris 8

Institut Universitaire de France

bvanruymbeke@univ-paris8.fr 